\title{
Des Amazones aux amazones. Équitation et statut féminin
}

\section{Catherine Tourre-Malen}

\section{(2) OpenEdition \\ 1 Journals}

Édition électronique

URL : https://journals.openedition.org/tc/1181

DOI : $10.4000 /$ tc. 1181

ISSN : 1952-420X

Éditeur

Éditions de l'EHESS

\section{Édition imprimée}

Date de publication : 1 décembre 2004

ISSN : 0248-6016

\section{Référence électronique}

Catherine Tourre-Malen, « Des Amazones aux amazones. Équitation et statut féminin », Techniques \& Culture [En ligne], 43-44 | 2004, mis en ligne le 15 avril 2007, consulté le 29 septembre 2022. URL:

http://journals.openedition.org/tc/1181 ; DOl : https://doi.org/10.4000/tc.1181

Ce document a été généré automatiquement le 29 septembre 2022.

Tous droits réservés 


\title{
Des Amazones aux amazones. Équitation et statut féminin
}

\author{
Catherine Tourre-Malen
}

1 Le mythe grec des Amazones ${ }^{1}$ nourrit non seulement les représentations de la femme à cheval mais encore celles du matriarcat. Les Amazones, cavalières d'excellence, vivent entre femmes et mènent la guerre contre le genre masculin. À l'égal des hommes, elles montent à califourchon, tirent à l'arc, occupent la sphère militaire... À l'inverse, la figure des amazones de l'Occident moderne -femmes montant obligatoirement, non pas à califourchon, mais les deux jambes serrées au contact du flanc gauche de l'animal - symbolise la grâce et l'«éternel féminin ». Que révèle la monte en amazone? Comment a-t-elle contribué à la hiérarchie et à la ségrégation entre les sexes ? Les réponses à ces questions permettront, peut-être, d'établir un rapport entre mythe et technique.

Le mythe des Amazones

2 La mythologie grecque présente les Amazones comme des filles de déesse (Aphrodite selon certains), réputées pour leur nature guerrière et pour avoir été les premières à recourir à la cavalerie (Graves 1999: 122). Ces femmes se caractérisent par des comportements masculins; elles pratiquent la ségrégation ou la soumission des mâles (Carlier-Détienne $1980: 12$ ). Amputées d'un sein, armées d'un arc et de flèches, elles montent à cheval, accomplissent des exploits, se battent comme des hommes et contre eux, vivent à l'écart des hommes, ou s'en servent comme esclaves. Dans certaines versions du mythe, ces sociétés ne reconnaissent que la filiation matrilinéaire: Lysippée, l'une des premières Amazones, ayant décrété que «les hommes seraient astreints à toutes les tâches domestiques, tandis que les femmes combattraient et gouverneraient» (Graves 1999 : 122), on brisait les membres des enfants mâles afin de les rendre inaptes au moins à la guerre et aux expéditions. D'autres versions évoquent une société sans homme: chaque printemps, les jeunes Amazones rencontrent des jeunes gens et s'unissent à eux. Les filles qui naissent de ces unions deviennent des Amazones; quant aux garçons, ils sont envoyés dans les tribus de leurs pères (Graves 1999 : 125). 
3 La découverte récente de tombes féminines contenant des armes et de défuntes dont les fémurs courbés indiqueraient une longue pratique de l'équitation donne à penser que les femmes guerrières ne relèvent pas entièrement de la fiction, et que le récit des Amazones "prend appui sur une réalité sociale pour raconter une belle histoire", transformant « une coutume minoritaire en une spécificité ethnique» (Testart 2002: $186,189)$. Ces femmes appartenaient à des sociétés des steppes (Scythes, Sarmates...) où il était assez fréquent qu'elles jouent un rôle militaire -en fait des peuples « spécialistes de la guerre » qui auraient fait une place aux femmes guerrières (Testart 2002 : 190, 192). Les femmes guerrières y étaient donc perçues comme une «réalité assez banale », alors que les Grecs firent des Amazones un thème récurrent de leur imaginaire (Testart $2002: 190)^{2}$ :

«S'il y a quelque gloire pour les héros grecs à se battre contre ces Amazones mythiques, il y en aurait guère pour les Scythes parce qu'ils ne se battraient que contre des femmes réelles. » (Testart $2002: 190)$

4 La haine des hommes, les communautés matriarcales et gynécocratiques des Amazones mythiques peuvent s'interpréter comme un pur fantasme, "engendré à coup d'inversions systématiques des valeurs et des normes de la société grecque » (Testart 2002: 187). Ce que dessinerait l'ensemble des traditions relatives aux Amazones, "malgré leurs hésitations entre un modèle d'opposition homme/femme d'une part, Grec/Barbare de l'autre ", c'est "une société où la femme est mâle, et vice-versa » (Carlier-Détienne 1980: 29). Le mythe aurait pour fonction de «montrer du doigt la monstruosité d'une société inversant les valeurs mâles» et de mettre en garde les hommes et leurs "dociles compagnes" contre «tout commencement de rébellion contre l'ordre mâle » (Carlier-Détienne $1980: 29$ ).

5 Pour les Grecs, les Amazones, qui avaient accès à la guerre et au cheval, étaient des créatures possédant un statut social et un pouvoir beaucoup plus élevés que ceux accordés aux femmes grecques, destinées à être au service des hommes (CarlierDétienne 1980 : 12). En leur donnant une dimension mythique, ils magnifiaient le courage de leurs héros masculins : les Amazones sont le plus souvent représentées au combat «saisies par les cheveux ou par le casque, ou pliant les genoux, la gorge transpercée de lances », c'est-à-dire comme une "race vaincue » (Van Creveld 2002 : 71).

6 Dans la mesure où il cherche à la fois à dénoncer une "société-monstre " (CarlierDétienne 1980 : 30) et à glorifier le courage des hommes, le mythe des Amazones paraît à l'origine plus masculin que féminin. Il fait pourtant référence au féminin; et les travaux d'Alain Bertrand (2000) montrent qu'au fil des siècles, aux figures mythiques dont ont parlé Homère, Hérodote et d'autres anciens, sont venues se superposer les héroïnes de la Renaissance, celles de l'époque romantique, puis les Amazones modernes des romans féministes, des romans noirs ou de science-fiction. En comparant les différentes versions du mythe, Alain Bertrand dégage trois points.

7 a) Des invariants (segments narratifs communs à plusieurs occurrences du mythe) : les différentes versions font état de femmes, barbares (au sens grec d'étrangères), indépendantes sexuellement, socialement, politiquement et qui n'utilisent les hommes que lorsqu'elles en ont besoin;

8 b) des mythèmes (fragments narratifs, englobant selon la définition de Levi-Strauss les invariants): l'analyse des textes relatifs aux Amazones d'Asie (Hérodote), d'Afrique (Diodore), d'Amérique (Fernández de Oviedo) et d'Europe (Æneas Silvius) révèlent des 
mythèmes narratifs s'attachant au mariage, au statut des hommes, au sort des enfants mâles, aux mutilations mammaires, à l'emploi du cheval, au combat, à la défaite et, enfin, au culte primitif de la déesse mère;

c) des attributs (éléments figuratifs ou symboliques indissociables) : le cheval, la hache bipenne (la sagaris ou labrys), et dans une moindre mesure le lys.

Se succèdent ainsi dans le temps: la "Tribale primitive", décrite par Hérodote, Diodore ou Starbon; la "Médiévale tyrannique", dont l'attitude se montre parfois radicalement féministe (Chrétien de Troyes); la guerrière de la Renaissance (Amazone belliqueuse de Boccace, féministe d'Agrippa, matriarcat d'Urfé); la "Révolutionnaire libertine ", qui participe aux grands bouleversements du XVIIe siècle; l' "Indépendante romantique » qui se caractérise par une volonté farouche d'autonomie et d'accès au pouvoir, et prend tour à tour l'aspect d'un garçon manqué contestataire (Théophile Gautier), d'une femme forte (Stendhal), d'une femme sans cœur (Balzac); enfin, la "Féministe d'après guerre". Cette dernière figure accompagne le combat des premières suffragettes et les théories féministes les plus radicales (Valérie Solanas).

11 Le mythe prend au XXe siècle une interprétation abrupte, déterminée tantôt par les mœurs des femmes qui se réclament des Amazones (Nathalie Barney, Renée Vivien), tantôt par la volonté et la violence militante (Monique Wittig, Françoise d'Eaubonne), en particulier des lesbiennes qui se sont lancées dans le combat féministe. Monique Wittig par exemple, «théoricienne du féminisme matérialiste », dénonce le mythe de la femme et l'hétérosexualité qu'elle définit comme la base d'un contrat social auquel les lesbiennes refusent de se soumettre ${ }^{3}$ : "N'étant femme "ni sémantiquement, ni politiquement, ni idéologiquement, [...], une lesbienne n'est pas une femme" " (Wittig citée par Deschamps $1989: 162$ ). Elle n'est pas non plus un homme, mais correspond à un « être inimaginable avec nos mots courants" (Deschamps 1989 : 162). Le mouvement lesbien ira jusqu'à brandir les attributs mythiques des Amazones, en particulier leur sagaris ou labrys, la hache bipenne (Bertrand 2000 : 179).

L'évolution contemporaine du mythe aboutit également à de nouvelles représentations de l'Amazone. Aux États-Unis, le terme Amazon « désigne, presque principalement, la femme physiquement puissante, donc tout d'abord la lutteuse musclée, mais aussi celle qui aime dominer l'homme, épouse despotique ou dominatrice professionnelle " (Bertrand 2000 : 179). En Europe, l'acception du mot reste plus féministe et met surtout sur l'accent sur la force et l'indépendance du caractère féminin. L'Amazone serait désormais « celle qui domine l'homme, dans quelque domaine que ce soit, car jamais un muscle, un uniforme ou un diplôme scolaire ne suffiraient par eux-mêmes à cette assimilation" (Bertrand 2000: 179). La prégnance des images véhiculées par les Amazones est telle qu'une féministe déclare : "La société des amazones a sans doute été plus favorable au développement du corps et des émotions des femmes qu'aucune société dominée par les hommes ne l'a jamais été » (Chesler 1972 : 286).

Il semble qu'à l'heure actuelle, le mythe des Amazones connaisse un renouveau inattendu porté par l'hypothétique existence d'un matriarcat primitif ${ }^{4}$.

«[le mythe des Amazones] au lieu de dire une vérité sacrée ou quelque proto-philosophie profane colportées illo tempore, ne [serait] conté que pour masquer un mythe premier oublié, [...] racontant une réalité antérieure, celle de la prééminence protohistorique et planétaire de la déesse et de la femme, celle du totalitarisme patriarcal établi par la force. La faillite actuelle de ce système patriarcal, lisible en particulier dans la crise écologique et 
politique du monde, aurait [...] un lien avec son instauration violente aux temps des commencements, tout comme sa probable fin que de nouvelles amazones devraient pouvoir hâter. » (Bertrand $2000: 4$ ) femmes d'aujourd'hui, de discours tenus par des mâles il y a deux mille ans (CarlierDétienne 1980 : 30), le mythe des Amazones reste vivant puisqu'il véhicule, comme autrefois, une charge idéologique.

\section{La monte en amazone}

siècle, un siècle qui connaît en France un véritable engouement pour le loisir équestre (Bouchet 1993 : 283). Le cheval, alors omniprésent dans la vie de la haute société, représentait " un symbole, un état d'esprit, une légende, une utilité de tous les jours " (comtesse de Pange, citée par Bouchet 1993: 248). Les amazones appartenaient généralement à l'aristocratie ou à la grande bourgeoisie, et montaient à cheval par "goût sportif» (Nelson 1994: 103). La pratique de l'équitation présentait à cette époque la particularité d'un "dimorphisme technique » sexué. On trouvait, d'un côté, la monte à califourchon à l'usage quasi exclusif du sexe masculin, de l'autre, la monte en amazone, réservée et imposée aux femmes -en 1914, il eût paru « inconvenant de monter autrement » (Bouchet $1993:$ 258). en amazone exigeait d'etre bien assis, pas trop en avant, "la hanche droite reculée pour placer les deux épaules dans la même ligne, [...], la jambe droite tombant naturellement et sur l'avance de la selle [...] la jambe gauche [...] pliée et appuyée sur la selle, ni tendue en avant, ni recourbée en arrière » (Pellier 1987 : 175). Une cravache tenue dans la main droite remplaçait l'action de la jambe droite. Ainsi placées, les femmes sont censées pouvoir encaisser, non seulement les secousses des allures vives, mais encore les écarts, les bonds, les sauts et les cabrades (Pellier 1987 : 111). La posture en amazone était considérée comme féminine par excellence :

«En équitation, la grâce est tout entière dans le charme de la position qui fait la femme si légère qu'on dirait qu'elle plane sur sa monture comme une gazelle, plutôt qu'elle n'y est retenue, et que toujours elle est prête à en descendre pour toucher terre en un froufrou délicat et charmant. » (Pellier in Anonyme $1918: 213$ )

répondre aux canons de la féminité du XIXe siècle, la femme à cheval devait se garder d'être trop hardie: «L'amazone [...], malgré l'origine belliqueuse de son nom, n'est pas obligée de nos jours, à faire preuve de force physique, d'audace et de témérité » (Pellier 1861 : 127).

En 1986 encore, cette position symbolise la féminité: le lieutenant-colonel de Beauregard, écuyer en chef du Cadre noir de Saumur, préfaçant L'Équitation en amazone, exprime la double reconnaissance des hommes - «en tant que cavaliers d'abord, mais aussi en tant que perpétuels amoureux du merveilleux féminin »- envers l'auteur pour leur avoir rendu « les amazones de [leurs] rêves » (Beauregard 1986 : 10).

Bien qu'élégante, cette façon de monter ne donnait qu'une autonomie relative et limitait la pratique de l'équitation. L'encombrement des jupes entravait les gestes des cavalières, si bien que se mettre à cheval et en descendre exigeait un apprentissage, non seulement de la part de la dame, mais de l'homme qui l'assistait. Il était d'ailleurs déconseillé pour cela de s'adresser au premier venu « sous peine d'être ridicule ainsi que lui » (Anonyme 1918: 207). La dame devait prendre appui sur l'épaule du cavalier qui se tenait en face d'elle, le corps légèrement incliné et les mains croisées un peu au- 
dessus des genoux pour recevoir le pied gauche de la dame. Cette dernière se soulevait sur son pied droit puis, soutenue par les mains croisées qui lui servaient d'étrier et appuyant fortement la main gauche sur l'épaule du cavalier, elle s'enlevait, le corps droit, et s'asseyait sans à-coup, doucement et à son aise (Vergnaud s. d. [1842] : 191). Pour mettre pied à terre, une fois l'étrier déchaussé, la dame, la poitrine tournée vers la selle, se laissait doucement glisser jusqu'au sol, le corps droit et la taille soutenue entre les mains du cavalier (Vergnaud s. d. [1842] : 195). Comme le constate une cavalière de cette époque, «il est presque impossible de remonter sans secours sur une selle de dame » (Archives nationales $1906: 145)$. Aussi est-il recommandé qu'« un cavalier, aide indispensable pour qu'une dame puisse monter à cheval et en descendre commodément, l'accompagne dans toutes ses promenades; il doit être assez bon écuyer pour donner, en toute circonstance, le précepte et l'exemple des principes d'équitation» (Vergnaud s. d. [1842] : 189). La posture des amazones les mettait dans un équilibre précaire qui imposait qu'elles ne montent que des chevaux sages :

«Un cheval ne peut convenir aux leçons de manège pour les dames, s'il n'est parfaitement sûr et solide des jambes de devant, d'une entière docilité à la main, sans humeur ou excès de sensibilité, habitué aux aides de la cravache, calme et d'une sagesse imperturbable au milieu des autres chevaux; enfin, dressé soigneusement, exprès, avec la selle à fourches, à des allures franches et très régulières. » (Vergnaud s. d. [1842] : 187)

Baucher, un écuyer du XIXe siècle ${ }^{5}$, va jusqu'à penser que, non seulement la position des femmes, mais encore leur tempérament restreignent la possibilité de contrôler un cheval :

«Une dame peut devenir très bonne cavalière, c'est-à-dire mettre facilement son cheval à toutes les allures, le conserver en équilibre et le conduire avec précision mais elle doit prendre le soin de ne monter qu'un cheval sage et bien dressé; d'abord parce qu'il n'est pas dans le caractère de ce sexe timide autant que gracieux de s'exposer à batailler avec son cheval; ensuite parce que celui-ci ne tarderait à s'apercevoir du peu de force qu'on lui oppose, et que d'ailleurs la position d'une femme ne comporte pas assez de moyens de répression. » (Baucher 1851, cité par Cabaud $1986: 87$ )

21 Molier ${ }^{6}$ quant à lui, ne rapporte l'infériorité des moyens d'action de l'amazone qu'à sa position; «Si [...] la femme sait monter en homme, il n'y a pas de raison qu'elle ne réussisse pas aussi bien que lui » (cité par Cabaud 1986: 260). La monte en amazone fragilise si bien qu'en cas de problème, la chute paraissait inévitable :

«Les femmes, sur nos selles modernes, quoique très confortablement installées, n'ont pas de soutien du côté droit. Tant qu'elles sont placées en face de leur cheval, tout est bien; mais si un violent bond de surprise les fait glisser à droite, infailliblement elles tombent; rien ne peut les empêcher. » (Pellier 1987 : 181)

Les plus grandes précautions étaient recommandées pour s'initier à la pratique de l'équitation car la chute paraissait « beaucoup plus dangereuse pour la femme que pour l'homme » : un cavalier déséquilibré pouvait se laisser glisser le long de l'encolure où il se raccrochait plus ou moins, tandis que l'amazone tombait « infailliblement » sur la tête (Anonyme $1918: 204$ ).

23 Si la "fragilité » de l'amazone autorisait une exploitation, parfois même virtuose, de montures dressées, elle ne permettait guère, compte tenu du peu de moyens de coercition et de stabilité qu'elle offrait, de dresser un cheval, c'est-à-dire de le conditionner aux actions du cavalier ${ }^{7}$. Le dressage fait partie de ces savoir-faire techniques spécialisés, à l'usage exclusif du sexe masculin, qui nécessitent «un 
apprentissage réellement ou faussement sophistiqué, mais dont rien, dans la constitution féminine, n'explique que la femme n'y ait pas accès » (Héritier 1996 : 233). Les limites techniques engendrées par la monte en amazone expriment une répartition sexuelle des tâches; elles révèlent, au sein d'une activité accessible aux deux sexes, l'existence d'un "domaine réservé " masculin (Héritier 1996: 234). Les très rares femmes qui prétendaient à ce domaine débordaient du strict espace qui leur était consenti en matière d'équitation (Houbre 2003: 46). Pour ce motif, Marie Isabelle - «inventrice » d'une méthode de dressage à pied achetée par le ministère de la Guerre en 1875 , le surfaix-cavalier- se trouva brocardée par les cavaliers de l'époque, en majorité des militaires; sa méthode, présentée comme une "recette de bonne femme " dont le fond est constitué par l'utilisation de morceaux de sucre et les carottes, fut tournée en ridicule (Vaux 1893: 56). La dresseuse, percevant combien son sexe fait obstacle dans une société peu « encline à admettre qu'une femme puisse conceptualiser une méthode de dressage ", revendiquera en vain "l'effacement de son sexe derrière son activité professionnelle » (Houbre $2003: 172$ ).

La marque de l'aliénation

L'approche diachronique de l'« équitation des dames » montre que pendant plusieurs siècles, les femmes pouvaient aller à cheval soit à califourchon, soit en amazone; mais les coutumes ont marginalisé la première manière et généralisé la seconde dont l'apogée se situera à la fin du XIXe siècle. Les perfectionnements des harnachements et de la technique visent à offrir aux femmes la plus grande stabilité possible, tout en les préservant d'ouvrir les cuisses pour chevaucher. La position du cavalier à califourchon implique une intimité troublante : le cavalier, assis, jambes écartées, absorbe, par le jeu de son corps, les mouvements de sa monture. Pour "accepter» le cheval, il lui faut ouvrir les cuisses sans contraction en relâchant ses muscles adducteurs appelés aussi "gardiens de la virginité » (Bourlet 2001 : 134). C'est à ce prix qu'il peut encaisser les secousses des allures mais aussi communiquer avec sa monture -l'idéal se situant dans une adhérence totale et une communication si fine qu'elles entraînent la "fusion " cavalier-cheval. Cette position prend chez les femmes une dimension particulièrement intime et sensible compte tenu des zones concernées, qui se trouvent de ce fait soumises, selon certains, à une "titillation particulière» (Bourlet 2001: 134). En amazone, jambes "parallèles », les femmes observent une décente "neutralité ». Cette technique, en évitant tout contact intime, empêche la fusion avec l'animal et dissocie la femme du cheval. L'amazone paraît flotter au-dessus de sa monture, rendant ainsi la monte "immaculée " (Saumade 2004). La dimension "protectrice» de la monte en amazone est connue du XIXe siècle; ainsi un voyageur oriental à Paris en 1860, qui demande des éclaircissements sur la manière particulière des femmes de monter à cheval, s'entend répondre: "C'est qu'elles ont quelque chose de précieux qu'elles craindraient de déchirer si elles ouvraient les jambes » (Al'Amraoui $2002: 86$ ).

Mais l'interdiction de chevaucher ne s'attache pas qu'à l'idée de préservation physique. Le chevauchement est aussi symbole de pouvoir : pouvoir des hommes sur les femmes par le biais de la sexualité, pouvoir des hommes sur le cheval et sur les autres hommes par le cheval. Selon certains mythes (Godelier 1982) qui établissent « les principes de la bonne sexualité et de l'ordre du monde» (Bozon 2001: 171), la femme prenait l'initiative dans les actes sexuels originels: elle apprenait à l'homme comment faire l'amour et se plaçait au-dessus de lui pendant le coït. Mais une fois le bon ordre social instauré et la sexualité "réglée", c'est l'homme qui chevauche la femme. Ce retournement de la situation permet aux hommes de contenir et de domestiquer les 
femmes. Le fait qu'ils occupent une position supérieure lors de l'acte sexuel justifie, toujours selon ces mythes, qu'ils doivent gouverner: "Imaginer un monde où les femmes chevauchent les hommes serait aussi absurde qu'imaginer un monde social où les femmes gouvernent»(Bozon 2001: 171). Dans l'Antiquité, celui qui se laisse chevaucher par une femme est un «impudique " (Bozon 2001: 175). Les théologiens médiévaux reprennent également l'idée d'une «bonne sexualité » qui respecte l'ordre du monde en recommandant une position dite «naturelle», l'homme par-dessus la femme : "Lorsque l'homme est en dessous, il subit, par le fait même de cette position, et lorsque la femme est dessus, elle agit. Combien la nature a horreur de cette mutation, qui ne le voit?» (Bozon 2001 : 172, citant Sanchez 1607). Cette idée perdure jusqu'au XIXe siècle où l'acte sexuel pratiqué la femme au-dessus de l'homme, considéré comme « la position inverse de celle qui est dite normale » (Bozon 2001 : 179), est interprété comme "une forme de masochisme masculin ou de sadisme (voire d'homosexualité) de la femme » (Krafft-Ebing 1886, cité par Bozon 2001 : 179).

La notion de domination s'étend à la pratique de l'équitation. Le cheval, symbole privilégié du pouvoir de l'homme sur l'animal, est perçu comme «l'emblème et l'instrument d'un autre pouvoir, celui de l'homme sur l'homme » (Digard 1999 : 56). Par ailleurs, le rôle dévolu à la théorie du dressage tend à conférer au cheval une dimension politique : "Apprendre à monter à cheval, c'est apprendre à gouverner son peuple " (Grange 1988: 66). En Occident, la relation entre l'art de la guerre et l'art de la cavalerie place le cheval au centre d'un "culte immémorial » en tant que "piédestal mouvant du gentilhomme » (Houbre $2003: 15$ ). Les premiers titres de la noblesse et de l'armée sont intimement liés au cheval : «Servir l'Église et l'État à la fin du Moyen Âge, c'est d'abord très concrètement pouvoir monter à cheval» (Grange 1988: 64). Ce « système de valeurs de distinction sociale, économique et militaire » (Grange 1988:63) généré par le cheval et plus particulièrement l'équitation, entretient également la ségrégation des sexes :

«L'utilisation du cheval à des fins guerrières, les compétences et la disponibilité de temps nécessitées par l'élevage et le dressage de cet animal puissant et fougueux ont contribué à forger, au fil des siècles, un cavalier archétypal aux traits aristocratiques et/ou militaires, masculins en tout cas. » (Digard 1999 : 57)

Empêchées d'ouvrir les cuisses pour chevaucher, les femmes se trouvent doublement écartées du pouvoir, du pouvoir sur l'animal et du pouvoir par l'animal. Il est possible d'établir un parallèle entre cet accès particulier des femmes au cheval (avant le XXe siècle) et leur accès aux outils. Les femmes se voient "constamment refuser la possibilité de prolonger leur corps et leurs bras d'outils complexes qui augmenteraient leur pouvoir sur la nature » (Tabet $1998: 68$ ); de même en équitation, on leur refuse une technique qui leur garantirait un contrôle performant de l'animal et leur donnerait un accès complet au monde équestre. L'étude de la différenciation sexuelle des outils dans certaines sociétés primitives met en évidence que le travail féminin est bloqué par deux interdits: l'emploi des armes et des outils-armes, et l'emploi des instruments de production complexes (Tabet 1998 : 57). Ces interdits assurent aux hommes un contrôle de tout le processus productif et confinent les femmes dans des activités subordonnées, même lorsqu'elles accomplissent la plus grande partie des opérations agricoles. Le double rôle des outils masculins explique aussi leur "rôle symbolique, leur identification avec le sexe masculin ou avec la virilité " (Tabet 1998: 57). L'accès à l'équitation suit la même logique : les femmes ont un accès au cheval via une technique qui les met dans une position handicapante et les « confine dans un rôle subordonné ». 
La maîtrise de l'équitation permet aux hommes d'asseoir leur pouvoir et, corollairement, de construire une dimension symbolique masculine qui contribue à la hiérarchisation entre les sexes.

À la fois signe de, et contribution à une «production sociale du genre » (Zaidman 2002 : 26), la monte en amazone participe à la catégorisation hiérarchisée des sexes. Elle témoigne du contrôle du corps des femmes et collabore à l'usage social qui en est fait un usage qui contribue à la construction sociale et influence l'ordre du monde. La gestion de la virginité, l'exaltation de la chasteté, les nouvelles manières d'un corps dompté, dissimulé, redressé, tout comme l'émancipation qu'illustrent le rejet du corset, le port du pantalon ou l'apparition du sport féminin représentent des éléments constitutifs du monde social ${ }^{8}$. La monte en amazone fait partie des «coutumes et modes " qui se sont "appliquées à couper le corps féminin de sa transcendance » au même titre que « la chinoise aux pieds bandés », « les griffes de la star d'Hollywood » ou « les talons hauts, les corsets, les paniers, les vertugadins, les crinolines, destinés moins à accentuer la cambrure du corps des femmes qu'à augmenter l'impotence " (Beauvoir 1999 : 205). Cette monte révèle comment une «technique du corps » (Mauss 1966 : 363) collabore à l'aliénation des femmes; ces dernières, rendues dépendantes des hommes, non seulement ne se révoltent pas contre une pratique contraignante du cheval mais la pérennisent. On retrouve un phénomène similaire concernant le port du corset: malgré les propos alarmistes des hygiénistes, les mères elles-mêmes veillent à façonner la taille de leur fille «en la coinçant, dès la puberté, dans les mâchoires de l'étau redoutable » (Perrot $1984:$ 173). Ce processus, ces « corps immolés » (Perrot $1984: 157)$, révèlent «l'existence d'un consentement des dominés à leur domination, et de dispositifs sociaux et idéologiques pour créer ce consentement », conséquence d'un "dispositif social qui légitime les droits et les devoirs inégaux de chaque sexe » (Godelier 1982 : 60).

Les amazones du XIXe siècle, réduites à une forme d'impotence afin de répondre aux convenances d'une société où les normes s'élaborent sur la ségrégation sexuelle des tâches, l'« asymétrie des paraîtres » et le " dimorphisme vestimentaire » (Perrot 1984 : 163), se trouvent très éloignées des représentations féminines véhiculées par le mythe des Amazones. On peut s'étonner de l'indifférence de ces femmes à la désaliénation : comment ont-elles pu se plier sans réagir à ce modelage, à cette appropriation de leur corps? La reproduction d'une norme, si curieuse ou révoltante soit-elle, tient à la fonction sociale essentielle qu'assument les représentations. Façonnées par la culture, ces dernières engendrent une vision du monde particulière où la perception se fait selon des conventions bien précises - la culture « désignant les stimuli à percevoir, tout en y associant symboles et valeurs (positives et négatives) et en y prescrivant le type d'expression émotionnelle qu'il convient d'adopter face à eux » (Vinsonneau 1997 : 80). Pour que les femmes abandonnent la monte en amazone, il a fallu que l'univers culturel se modifie; il a fallu attendre que les «choses deviennent pensables c'est-à-dire acceptables de façon majoritaire $"^{9}$-ce qui advient légalement en 1930 , année où le règlement des concours hippiques précise que «les dames sont admises à monter à califourchon $»^{10}$. Mais on peut penser que «les faits ont précédé le droit » (TourreMalen s/presse). Désormais les femmes accèdent au même statut que les hommes dans l'apprentissage de l'équitation ${ }^{11}$. Notons que cet accès égalitaire ne se réalise qu'une fois que l'équitation a quitté la sphère militaire -la cavalerie apporte une première fois à Sedan, puis définitivement en 1914-1918, la « démonstration de son incapacité à jouer 
un rôle décisif dans les batailles " (Digard 2002 : 103) - pour entrer dans la sphère sportive. Les femmes en profiteront massivement : en 2002, les cavalières représentent plus de $70 \%$ des licenciés de la Fédération française d'équitation.

Des Amazones se dégagent donc deux figures féminines symétriques et inverses: la première symbolise une femme égale, alter ego, de l'homme, plus encore, sa rivale, qui fût l'icône des mouvements féministes et des organisations radicales de l'émancipation féminine et lesbienne; la seconde, associée à la «femme à cheval " exprime, via une technique qui la distingue du masculin, l'essence d'une féminité façonnée par la grâce et les convenances, aliénée en fait, la technique étant le vecteur de cette aliénation. D'où procède cette technique? Il est tentant d'avancer avec Lederer (1970): de la "peur latente de la femme ", profondément ancrée dans la psyché masculine. Effrayés par les Amazones mythiques, ces femmes-monstres aux traits aberrants, les hommes auraient limité l'accès des femmes au cheval en élaborant une " frontière " technique. Pour cela, ils auraient « simplement » mis en application les propos d'Aristophane :

«Si l'un d'entre nous leur [les femmes] cède et leur donne la moindre prise, il n'est rien que ces [gaillardes] n'entreprennent de leurs mains tenaces [...], si elles se tournent vers l'équitation, je biffe nos rôles de cavalier. Car comme cavalière, la femme excelle et se tient ferme; elle ne glisse point même au galop. [...]. Allons, il faut nous assurer de toutes et adapter un carcan à tous ces cous-là. » (Aristophane, Lysistrata, cité par Van Creveld $2002: 189$ )

La monte en amazone, le "carcan", ne résistera pas à la modernité et au processus irréversible qui voit, au tournant des XIXe et XXe siècles, l'intrusion de femmes dans des domaines jusque-là masculins. Cette intrusion tend à prouver que les activités investies ne conféraient pas de «brevet de masculinité " par leur contenu mais "seulement par l'interdiction faite aux femmes de les pratiquer" (Maugue 2001: 65). Et si elle n'implique pas d'égalité entre les femmes et les hommes, elle montre, en revanche que les femmes peuvent exercer le même métier ou maitriser la même technique que les hommes et remporter les mêmes succès qu'eux, renouant ainsi avec le fondement du mythe des Amazones.

Cette recherche s'inscrit dans le cadre d'une convention CNRS/Haras nationaux, en partenariat avec la Commission européenne (Fonds social européen).

\section{BIBLIOGRAPHIE}

Al'Amraoui, Idriss

2002. Le Paradis des femmes et l'enfer des chevaux. La Tour d'Aigues : L'aube (« L'aube de poche »).

Anonyme

1918. Pour bien faire du sport. Paris : Pierre Lafitte \& Cie (« Fémina Bibliothèque »).

Archives nationales

1993. Les Sources de l'histoire du cheval dans les archives publiques françaises. Paris. 
Bertrand, Alain

2000. L'archétype des amazones. Thèse de doctorat de l'Université Paris 4 (sous la direction de Pierre Brunel).

Beauregard (de), lieutenant-colonel

1986. « Préface », pp. 9-10, in Madame Henry Cabaud, L'équitation en amazone. Paris : Crépin-

Leblond.

Beauvoir (de), Simone

1999. Le Deuxième sexe. Paris : Gallimard (« Folio ») (1ère éd. 1949).

Bouchet, Ghislaine

Le Cheval à Paris de 1850 à 1914. Genève-Paris : Librairie Droz (« Mémoires et documents de l’École des Chartes »).

Bourlet, Dominique

2001. « Le hussard et la jeune fille », L'Internationale de l'Imaginaire 14 (numéro spécial « Eros et Hippos ») : 133-139.

Bozon, Michel

2001. « Sexualité et genre », pp. 169-186, in Jacqueline Laufner, Catherine Marry et Margaret

Maruani (eds), Masculin-Féminin : questions pour les sciences de l'homme. Paris : Presses

Universitaires de France (« Sciences sociales et société »).

Cabaud, Madame Henry

1986. L'équitation en amazone. Paris : Crépin-Leblond.

Carlier-Détienne, Jeannie

1980. « Les Amazones font la guerre et l'amour », L'Ethnographie XXVI, 81-82 : 11-33

Chesler, Phyllis

1997. Women and Madness. New York-London : Four walls eight windows.

Deschamps, Gaële

1989. « Fragments d'une mémoire lesbienne », pp. 135-191, in J. Decottignies (ed.), Physiologie et mythologie du « féminin ». Lille : Presses Universitaires de Lille.

Digard, Jean-Pierre

1999. Les Français et leurs animaux. Paris : Fayard.

2001. «Sociologie de la relation homme-cheval », pp. 472-475, in

J.-F. Charry (ed.), Encyclopédie du cheval. Paris : Aniwa Publishing.

2002. «Équitation militaire et équitation académique : histoire et enseignements d'un

antagonisme pluriséculaire », pp. 95-107, in D. Roche (ed.), Le Cheval et la guerre. Paris : Association pour l'académie d'art équestre de Versailles.

Godelier, Maurice

1982. La Production des Grands hommes. Pouvoir et domination masculine chez les Baruya de Nouvelle-Guinée. Paris : Fayard.

Grange, Yves

1988. « Signification du rôle politique du cheval (XVIIIe et XIXe siècle), pp. 63-70, in J.-P. Digard

(ed.), Des chevaux et des hommes. Équitation et société. Lausanne : Favre.

Graves, Robert

1999. Les Mythes grecs. Paris : Hachette Littérature (« Pluriel ») (1ère édition 1958 : Robert Graves).

Héritier, Françoise

1996. Masculin/féminin. La pensée de la différence. Paris : Odile Jacob. 
Houbre, Gabrielle

2003. Grandeur et décadence de Marie Isabelle, modiste, dresseuse de chevaux, femme d'affaires, etc. Paris : Perrin.

Lederer, Wolfgang

1970. Gynophobia ou la peur des femmes. Paris : Payot.

Marry, Pascal

2002. «D’une pédagogie héritée à une pédagogie choisie », pp 67-85, in Apprendre le cheval autrement, Colloque de novembre 2001 à l'École Nationale d'Equitation Paris : Belin.

Maugue, Annelise

2001. L'Identité masculine en crise au tournant du siècle 1871-1914. Paris : Payot (« Petite bibliothèque Payot ») (1ère édition 1987).

Mauss, Marcel

1966. « Les techniques du corps », pp. 363-386, Sociologie et Anthropologie. Paris : Presses

Universitaires de France (1ère édition 1936).

Nelson, Hilda

1994. «L'amazone et l'écuyère au XIXe siècle », pp. 101-113, in De Pégase à Jappeloup. Cheval et société. Festival d'histoire de Montbrison : Ville de Montbrison.

Pellier, Jules

1861. L'Équitation pratique. Paris : Hachette.

1987. La Selle et le costume de l'amazone. Paris : Jean-Michel Place (1ère édition 1897).

Perrot, Philippe

1984. Le Travail des apparences. Le corps féminin XVIIIe-XIXe siècle. Paris : Le Seuil (« Points »).

Saumade, Frédéric

2004. « Animal de rente et animal de loisir : l'énigme de la monte du taureau au Mexique »,

Anthropozoologica 39 : 61-72.

Tabet, Paola

1998. La Construction sociale de l'inégalité des sexes. Paris : L'Harmattan.

Testart, Alain

2002. « Amazones, mythe et réalité », L'Homme 163 : 185-194.

Tourre-Malen, Catherine

s/presse. « Des amazones aux cavalières : avatar d'un loisir féminin », in D. Roche (ed.), Le Cheval et les loisirs (XVIe-XXe siècle). Paris : Association pour l'académie d'art équestre de Versailles.

Van Creveld, Martin

2002. Les Femmes et la guerre. Paris : Éditions du Rocher (« L'art de la guerre »).

de Vaux, Baron

1893. Écuyers et écuyères. Histoire des cirques d'Europe. Paris : Rothschild.

Vergnaud, A. D

s. d.. Le Nouveau manuel complet d'équitation à l'usage des deux sexes. Paris : Manuels Roret. (2ème edition 1842, réédition : Paris : C. Baudoin, s. d).

Vinsonneau, Geneviève

1997. Culture et comportement. Paris : Armand Colin. 
Zaidman, Claude

2002. « Ensemble et séparés », pp. 9-37, in E. Goffman (ed.), L'Arrangement des sexes. Paris : La Dispute.

\section{NOTES}

1. On parlera ici du « mythe des Amazones » bien que l'étymologie du terme « amazone » pose de nombreuses questions non résolues (Bertrand 1999:10) et que certains contestent l'intitulé du récit, préfèrant parler de mythe de la femme virile (Boyer 1988), de la femme phallique ou de la guerre des sexes (Gliksohn 1997).

2. La réalité des femmes guerrières renvoie aux « configurations sociales » qui caractérisent respectivement les «peuples cavaliers » et les « sociétés à écuyers » (Digard 2001 : 472). Chez les peuples cavaliers, comme les nomades d'Asie centrale, «tous les membres, hommes, femmes et enfants, montent peu ou prou à cheval »; cet animal y envahit tout le « champ social ou culturel » (Digard 2001 : 472). Dans les " sociétés à écuyers ", comme la société occidentale, l'équitation est " réservée à une minorité, par nécessité de métier et de fonction ou par privilège de classe » (Digard 2001 : 473). Le cheval revêt alors « une valeur d'emblème que protègent de nombreuses prohibitions » (Digard $2001: 474)$, et l'équitation joue une fonction distinctive.

3. Le Monde du 11 janvier 2003.

4. Hypothèse émise en 1861 par Johann Jakob Bachofen (cité par Bertrand 2000 : 156).

5. Auteur de plusieurs traités d'équitation dont un Dictionnaire raisonné de l'équitation (1833) et des Dialogues sur l'équitation (1888).

6. Écuyer réputé des années 1900, directeur de cirque et auteur de L'Équitation et le cheval.

7. Le conditionnement d'un cheval, son dressage, correspond aux « apprentissages qu'il a effectués et qui organisent ses réponses et les rendent prédictibles par le cavalier » (Marry $2002: 81$ ).

8. Le Monde du 17 août 2001.

9. Françoise Héritier, Libération, 18 et 19 août 2001.

10. Le Club des Habits Rouges et les concours hippiques, 1931, circulaire adressée aux membres du club précisant les modifications importantes du règlement, article 12. 11. Au point même que l'équitation fait partie des très rares disciplines sportives totalement mixtes.

\section{RÉSUMÉS}

Le mythe grec des Amazones nourrit les représentations de la femme à cheval et celles du matriarcat. Ces cavalières d'excellence vivent entre femmes et mènent la guerre au genre masculin. À l'inverse, la figure des amazones de l'Occident moderne est la manifestation d'une mise en position d'infériorité et d'une ségrégation des sexes. La technique de la «monte en amazone ", qui met les femmes en équilibre précaire, est justifiée par les croyances liées à la 
nature féminine et à l'ordre social. La distinction entre hommes et femmes impose et résulte d'un «dressage» du corps féminin, intériorisé par les femmes elles-mêmes, au point qu'elles contribuent à le pérenniser. Le passage, dans les années 1930, de la monte en amazone à la monte à califourchon peut être interprété comme une avancée dans la voie de la désaliénation des femmes.

From Amazons to amazons. Equitation and the status of women.

The Greek myth of the Amazons sustains our ideas about not just women on horseback but also the matriarchate. The Amazons, an exemplar of women on horseback, live among women and wage war with men. In contrast, the figure of Amazons in the modern West is evidence that sexual segregation persists and that women are still assigned an inferior position. The technique of «riding like an amazon» (the French phrase for astraddle) places women in a precarious balance and limits their control over the horse. It is justified by beliefs related to the " feminine nature " and the social order. The distinction between men and women "breaks in " the woman's body, and is also its result thereof. This training is internalized by women to such a point that they help ensure its continuation. The shift during the 1930s from riding sidesaddle to riding astride can be interpreted as an advance in reducing the alienation of women.

De las Amazonas a las amazonas. Equitación y estatuto femenino.

El mito griego de las Amazonas alimenta tanto las representaciones acerca de la mujer jinete como aquellas en torno al matriarcado. Esas excelentes jinetes viven entre mujeres y hacen la guerra al género masculino. De manera inversa, la figura de las amazonas del Occidente moderno es manifestación de que las mujeres están en una posición de inferioridad, y que los sexos están segregados. La técnica de « monta a la amazona », que coloca a las mujeres en equilibrio precario, se justifica a partir de creencias ligadas a la naturaleza femenina y al orden social. La distinción entre hombres y mujeres impone y resulta en un « adiestramiento » del cuerpo femenino, que es interiorizado por las propias mujeres, hasta el punto de que ellas contribuyen a hacerlo perenne. El tránsito, en la década de 1930, de la monta a la amazona a la monta a horcajadas puede interpretarse como un avance en el camino de abolir la enajenación de las mujeres.

\section{INDEX}

Mots-clés : Antilles

\section{AUTEUR}

\section{CATHERINE TOURRE-MALEN}

Maître de conférences associé (Université Paris XII). c.tourremalen@wanadoo.fr 